\title{
Condiciones paleoambientales de sedimentación y preservación de materia orgánica en bahía Mejillones del Sur $\left(23^{\circ} \mathrm{S}\right)$, Chile*
}

\author{
Paleo-environmental sedimentation conditions and organic matter preservation \\ in Mejillones del Sur Bay (23 S), Chile. \\ Jorge Valdés ${ }^{1}$, Liliana López ${ }^{2}$, Salvador LoMónaco² y Luc Ortlieb ${ }^{3}$ \\ * Proyecto PALEOBAME (Convenio IRD-Universidad de Antofagasta), Proyecto FONDECYT 2960074 (Tesis doctoral). \\ ${ }^{1}$ Instituto de Investigaciones Oceanológicas, Facultad de Recursos del Mar, Universidad de Antofagasta, \\ Casilla 170, Antofagasta, Chile. \\ ${ }^{2}$ Instituto de Ciencias de la Tierra, Universidad Central de Venezuela, \\ Aptdo. 3895, Caracas 1010-A, Venezuela. \\ ${ }^{3}$ Institut de Recherche pour le Développement (IRD ex ORSTOM), Programme PVC, UR1, Centre de Recherche Ile de France, \\ 32 Avenue Henri-Varagnat F-93143, Bondy Cédex, Francia. \\ jvaldes@uantof.cl
}

\begin{abstract}
A combined study bearing on the downcore variations of metal elements and of organic matter composition in a $48 \mathrm{~cm}$-long sedimentary core provided elements to reconstruct paleoenvironmental conditions of oxygen availability and organic matter preservation in the Bay of Mejillones del Sur $\left(23^{\circ} \mathrm{S}\right.$, northern Chile).

High concentration of heavy metals like $\mathrm{Cd}$ and Mo, both indicators of former conditions of oxygenation, and the values of redox indices $\mathrm{V} / \mathrm{Cr}$ y Ni/Co in several points of the core, suggest a predominance of disoxic/anoxic conditions at the bottom of the water column all along the last millenium.

The transmission Fourier transformed infrared (FTIR) spectroscopy of the soluble organic matter showed that, during the last thousand years, the early diagenesis scarcely affected the organic components of the sediment: the spectra indicate that the functional groups are the same that those of the biological precursors (living organisms).

The high content of organic matter and its good state of preservation reflect the general lack of oxygen experienced by the subsuperficial waters of the embayment during the last millenium.
\end{abstract}

Key words: sediments, organic matter, paleoceanography.

\section{Introducción}

La condición redox, entendida como el estado de oxigenación de la zona de contacto entre la columna de agua y el sedimento, es la forma más práctica de caracterizar químicamente un ambiente de depositación marino. Normalmente el agua superficial se encuentra saturada de oxígeno, y a medida que aumenta la profundidad su concentración va cambiando debido a
Resumen.- El estudio combinado de metales y de materia orgánica soluble contenidos en un testigo de sedimentos de 48 $\mathrm{cm}$ de longitud ha permitido identificar las condiciones paleoambientales de sedimentación y preservación de materia orgánica en la bahía Mejillones del Sur $\left(23^{\circ} \mathrm{S}\right.$, norte de Chile). Concentraciones altas de metales como $\mathrm{Cd}$ y Mo, ambos indicadores de paleoxigenación, y el uso de los índices redox $\mathrm{V} / \mathrm{Cr}$ y Ni/Co a lo largo del testigo evidenciaron el predominio de condiciones de depositación disóxicas/anóxicas en el fondo de la bahía durante el último milenio.

El análisis IRTF realizado sobre la materia orgánica soluble y su fracción de hidrocarburos saturados evidenció que en el transcurso de los pasados mil años la diagénesis temprana ha afectado en grado mínimo a los constituyentes orgánicos del sedimento, ya que los espectros obtenidos mostraron que los grupos funcionales presentes en ellos son los mismos que normalmente pueden encontrarse en los precursores biológicos (organismos vivos).

El alto contenido de materia orgánica y su buen estado de preservación tienen relación directa con la pobreza de oxígeno que han presentado las aguas subsuperficiales de la bahía, durante el último milenio.

Palabras clave: sedimentos, materia orgánica, paleoceanografía. los procesos de remineralización de la materia orgánica y a las características propias de las diferentes masas de agua que allí se pueden encontrar (Libes 1992).

En el caso del sedimento, el oxígeno es suministrado sólo desde el agua inmediatamente en contacto con el fondo, y va disminuyendo con la profundidad debido a la descomposición bacteriana y a una limitada difusión (Brown et al. 1994). Esta situación genera tres zonas 
características que son definidas como zona óxica que abarca el $\mathrm{cm}$ superficial, zona subóxica de $1 \mathrm{a} 10 \mathrm{~cm}$ y zona anóxica ubicada entre los 10 y $100 \mathrm{~cm}$ (Alongi et al. 1996). Sin embargo, en centros de surgencia en donde ocurre un alto flujo de materia orgánica, el límite óxico- anóxico está desplazado hacia arriba, por lo que puede encontrarse incluso a una considerable distancia del piso marino (Didyk et al. 1978; Libes 1992; Brown et al. 1994).

Diversos autores (Didyk et al. 1978; Jones \& Manning 1994; Calvert et al. 1996) plantean que el rápido decaimiento de la concentración de oxígeno disuelto ejerce un fuerte control sobre la cantidad y calidad de la materia orgánica preservada en estos ambientes. Por otra parte, esta pobreza extrema de oxígeno inhibe el desarrollo de fauna bentónica, permitiendo una buena preservación de la estructura primaria de la columna de sedimento (Reineck \& Singh 1980). El registro sedimentario formado bajo estas condiciones ofrece una excelente oportunidad de estudiar la evolución del sistema océano-atmósfera a una escala geológica. Sin embargo, estas interpretaciones requieren de un claro conocimiento de las características pasadas del ambiente de sedimentación, especialmente su condición redox (Jones \& Manning 1994).

Si bien parte de la plataforma y talud continental de la costa norte-centro de nuestro país se ubica dentro de la zona de mínimo oxígeno (OMZ) de Perú y Chile (Kamykowski \& Zentara 1990; Gallardo 1992; Schrader 1992), no es fácil encontrar sectores con un buen registro sedimentario. Sin embargo, en el norte de Chile y precisamente donde la plataforma continental está prácticamente ausente, existe una pequeña bahía (Mejillones del Sur) con particulares condiciones geográficas y oceanográficas. Esta bahía, abierta hacia el norte y a sotavento de una gran península (Fig. 1) se encuentra protegida de las corrientes costeras de dirección norte, situación que favorece la existencia de un centro de depositación de abundantes restos biogénicos resultantes de la alta productividad de la zona.

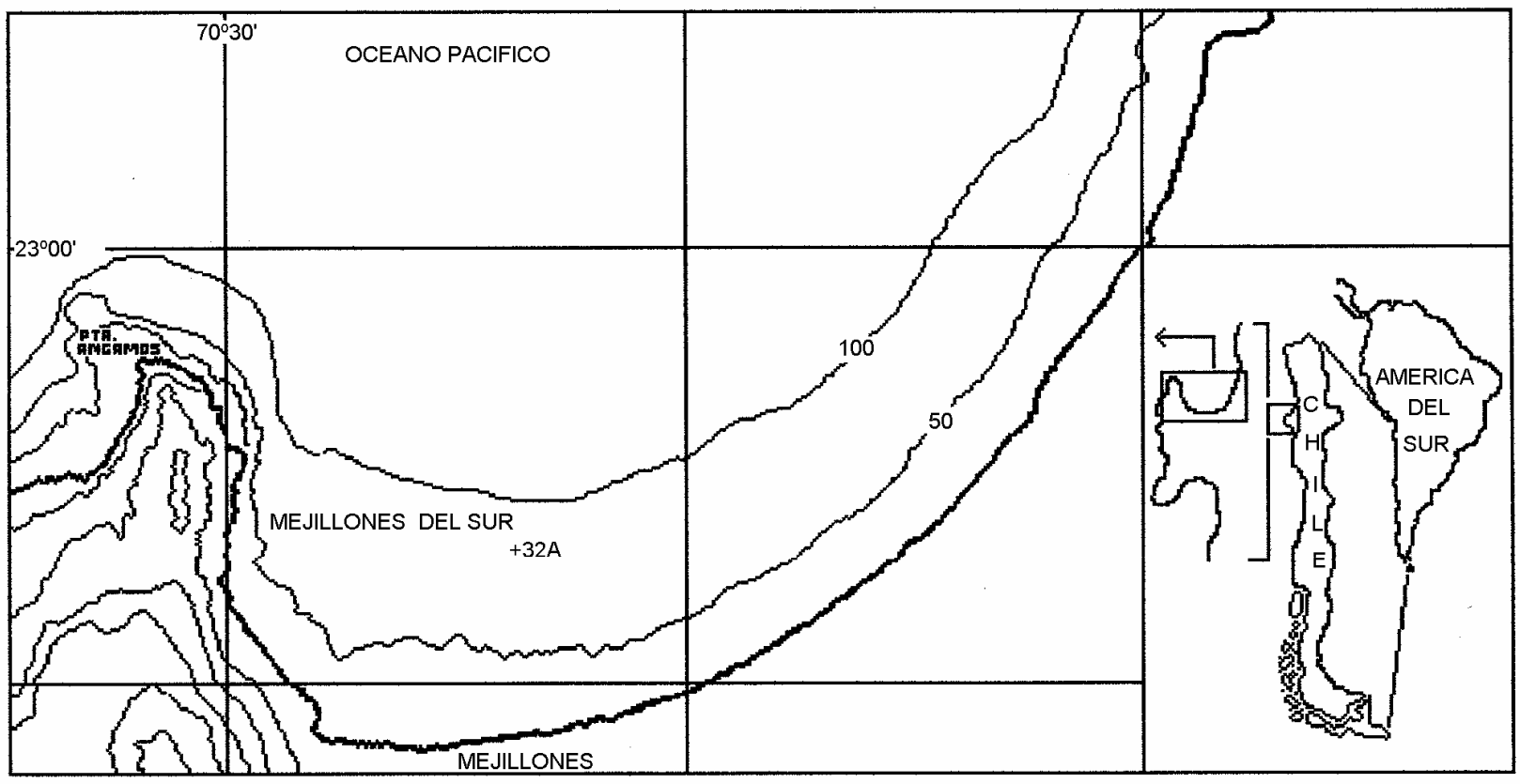

Figura 1

Situación geográfica de la bahía de Mejillones y ubicación del punto de muestreo a los $23^{\circ} 03$ ' $00^{\prime \prime} \mathrm{S}$ y 70 26 ' 56" W. La profundidad en el punto es de $93 \mathrm{~m}$.

Geographical situation of Mejillones Bay and location of the sampled core at $23^{\circ} 03^{\prime} 00^{\prime \prime} \mathrm{S}$ and $70^{\circ} 26^{\prime} 56^{\prime \prime} \mathrm{W}$. Water depth is $93 \mathrm{~m}$. 
Perfiles de oxígeno tomados en Mejillones durante el período 1994-95 (Fig. 2) muestran una capa superficial bien oxigenada $\mathrm{y}$ un brusco descenso de la concentración con valores inferiores a $0,3 \mathrm{ml} / 1$ desde los 50 $\mathrm{m}$ de profundidad. Por su parte, el límite de la capa anóxica $\left(\mathrm{O}_{2}\right.$ $<0,1 \mathrm{ml} / \mathrm{l})$ se ubica entre los 45 y $90 \mathrm{~m}$ de profundidad, dependiendo de la época del año.

Además del alto flujo de detritus orgánico y de las masas de agua presentes en la zona de Mejillones, existen otros factores que pueden intensifican esta condición al interior de la bahía. Por ejemplo, Reineck \& Singh (1980) y Jones \& Manning (1994) indican que el potencial redox está asociado a las condiciones hidrodinámicas, de tal manera que ambientes anaerobios sólo pueden mantenerse en ausencia de turbulencias y corrientes. Esta característica explicaría la existencia de sedimentos, en la parte media de la bahía, que han sido descritos como un "fango de color gris verdoso y olor pútrido" (Ramorino \& Muñiz 1970), rasgos propios de ambientes reductores (Reineck \& Singh 1980; Ridgway \& Price 1987).

Todos los antecedentes entregados más arriba muestran que en la actualidad la bahía presenta una condición redox típica de sistemas de surgencia, con una capa anóxica que comienza varios metros arriba de la interface agua-sedimento. Sin embargo, desde el punto de vista paleoceanográfico resulta interesante comprobar si estas condiciones también prevalecieron en el pasado.

Debido a que la productividad y las condiciones de oxigenación de la columna de agua son los factores principales que influyen sobre la calidad y cantidad de materia orgánica acumulada en los sedimentos marinos (Lallier-Verges et al. 1993; Hulthe et al. 1998; Ransom et al. 1998), es importante comprender cómo se han comportado estos factores a través del tiempo, y qué efectos han tenido sobre las características del registro sedimentario. Diferentes técnicas de estudio pueden ser empleadas para abordar estas interrogantes. Por ejemplo, el contenido de algunos metales traza en testigos de sedimento marino puede aportar al conocimiento de las condiciones de oxigenación de las aguas subsuperficiales en el pasado (Stein 1991; Jones \& Manning 1994; Calvert et al. 1996; Dean et al.
MES

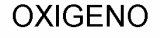

$M J J A S O N D$

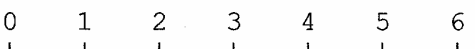

Figura 2

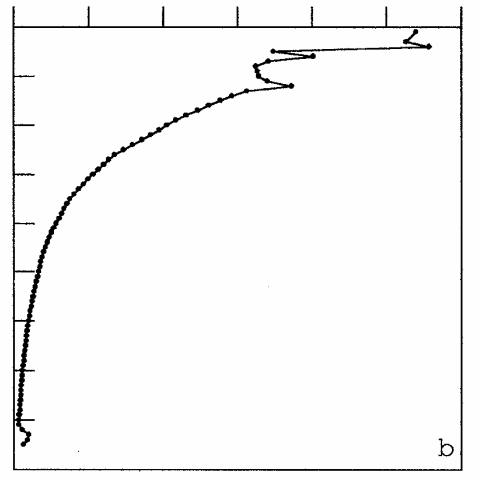

Oxígeno disuelto en la bahía Mejillones durante el período 1994-95. Datos no publicados proporcionados por Rubén Escribano, de la Universidad de Antofagasta.

Dissolved oxygen variability in Mejillones Bay in 1994-1995.

npublished data from Rubén Escribano, Universidad de Antofagasta.

1997). De igual forma, el estudio de la materia orgánica contenida en sedimentos anóxicos puede ayudar a comprender los efectos de la diagénesis sobre el registro orgánico, las características del ambiente de depositación (especialmente su condición redox), y la magnitud de la productividad de las aguas superficiales (Stein 1991).

Testigos de sedimento marinos tomados en distintos puntos de la bahía Mejillones han aportado importantes antecedentes sobre la evolución océano-climática de la zona durante los últimos milenios. Estos estudios, realizados sobre la base de bioindicadores, sugieren la ocurrencia de eventos cálidos tipo Mega El Niño y Episodio Cálido de la Edad Media, y de eventos fríos tipo Pequeña Edad del Hielo, que han quedado registrados en los sedimentos de la bahía (Ortlieb et al. 1994; 2000; Valdés, 1998).

El presente trabajo tiene como objetivo establecer las condiciones de sedimentación y preservación de materia orgánica en la bahía Mejillones, durante el último milenio, mediante el análisis de indicadores geoquímicos a lo largo de un testigo de sedimento.

\section{Materiales y Métodos}

Con un sacatestigos de gravedad tipo Phleger de 7,6 cm de diámetro se recuperó un testigo de $48 \mathrm{~cm}$ de longitud (32A), a $93 \mathrm{~m}$ de profundidad, en la parte media de la

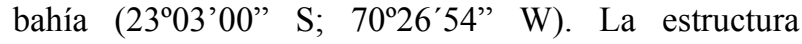
sedimentaria se describió mediante observación directa de la columna de sedimento y mediante una placa de 
radiografía por rayos $\mathrm{x}$ tomada con un equipo médico convencional (50 kv de potencia, 0,16 s de exposición, $1 \mathrm{~m}$ de distancia). Posteriormente el testigo fue cortado en secciones de $4 \mathrm{~cm}$ de alto, y los sedimentos fueron secados a $40^{\circ} \mathrm{C}$, por 48 horas.

La edad de este testigo fue estimada mediante la comparación de características sedimentológicas, con otros testigos cercanos en los que se realizaron mediciones de radiocarbono. Básicamente, el rasgo sedimentológico utilizado fue una discordancia angular en la orientación de los estratos sedimentarios que ha sido interpretada como el registro de un fuerte evento sísmico ocurrido en 1.200 años A.P. (Valdés 1998). La ausencia de esta discordancia en el testigo 32A permiten asignarle a la base de este testigo una edad de alrededor de 1000 años A.P.

Las concentraciones de Molibdeno (Mo), Cromo $(\mathrm{Cr})$, Cadmio (Cd), Vanadio (V), Níquel (Ni), Cobalto (Co) y Manganeso (Mn) se determinaron en un equipo de Plasma Secuencial marca JOBIN YVON JY24. Para el análisis se pesaron $4 \mathrm{~g}$ de sedimento y se les eliminó la materia orgánica mediante calcinación a $450^{\circ} \mathrm{C}$. Posteriormente los metales fueron extraídos mediante un ataque con $\mathrm{HNO}_{3}$ y $\mathrm{HCl}$ (1:3), a una temperatura de $100^{\circ} \mathrm{C}$, hasta que todo el ácido se evaporó, luego de lo cual se agregaron unas gotas de $\mathrm{HCl}$. Finalmente las muestras se filtraron (filtros milipore de $0,45 \mu \mathrm{m}$ ), se aforaron a $100 \mathrm{ml}$ y se determinó la concentración de los metales. Los resultados son entregados en ppm.

La caracterización de la materia orgánica se realizó en las muestras 0-4 cm, 12-16 cm, 20-24 cm, 32-36 cm y 44-48 cm. La extracción de materia orgánica se realizó en 2 g de sedimento seco de cada muestra, cantidad que se colocó en reflujo con cloruro de metileno $\left(\mathrm{CH}_{2} \mathrm{Cl}_{2}\right)$ en un equipo Soxhlet, por 5 horas. Posteriormente el extracto fue rotaevaporado para obtener la materia orgánica soluble en solvente orgánico (MOS)

La fracción de hidrocarburos saturados de la MOS fue separada por medio de cromatografía líquida de adsorción en columna, utilizando alúmina (100-120 mallas, activada a $200^{\circ} \mathrm{C}$ y $25 \mathrm{~mm}$ de $\mathrm{Hg}$ por 12 horas) como fase estacionaria. Posteriormente esta fracción fue eluida con n-Hexano $\left(\mathrm{C}_{6} \mathrm{H}_{14}\right)$.

Tanto la MOS como su fracción de hidrocarburos saturados fueron analizadas por espectrometría infrarroja (IR), utilizando un equipo Perkin-Elmer modelo 1760-X9, con un transformador de Fourier (IRTF). Para el análisis, las muestras fueron disueltas en cloruro de metileno y se analizaron en películas líquidas, utilizando ventanas de cloruro de sodio. El reconocimiento de los grupos funcionales se realizó según Silverstein et al. (1980) y Pretsch et al. (1989).

\section{Resultados}

La radioscopía $\mathrm{x}$ mostró en forma nítida una estructura laminar que dominó a lo largo de toda la columna de sedimento. La parte superior del testigo (Fig. 3) presentó una estructura homogénea, aún cuando pueden distinguirse delgadas láminas oscuras un tanto espaciadas. En la parte inferior las láminas claras y oscuras fueron mucho más nítidas y numerosas.

El análisis visual del testigo evidenció una alternancia de capas de solo 2 colores, 5 Y 4/4 y 5Y 5/6 (tabla de colores Munsell) (Fig. 3). El tercio superior presentó una estructura de color oscuro, mientras que el tercio inferior presentó una sucesión de delgadas capas claras y oscuras de entre 1 y $3 \mathrm{~cm}$ de espesor. Los contactos estuvieron bien definidos.

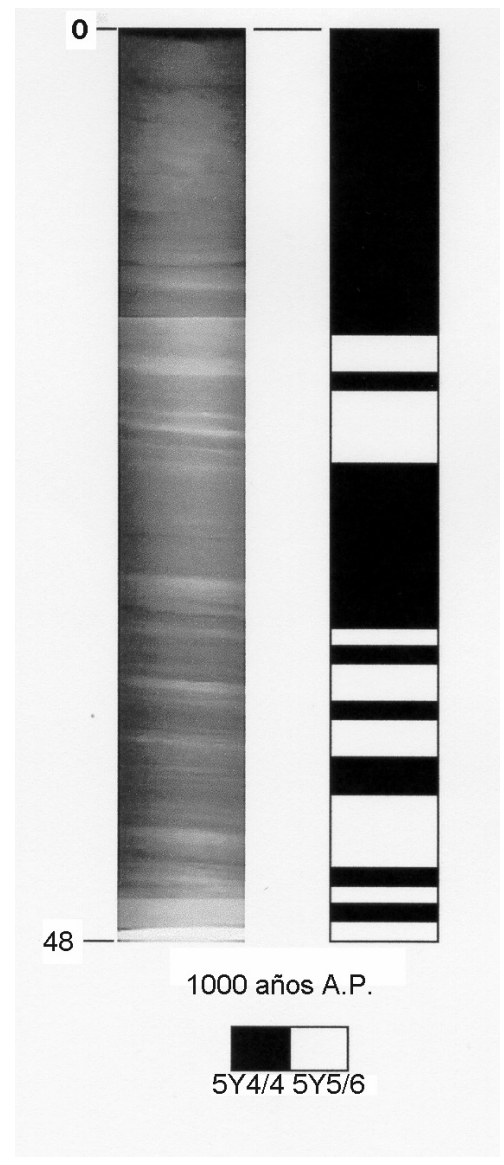

Figura 3

Estratigrafía del testigo 32A. Radioscopía $x$ (negativo) y tonalidades según tabla de Colores Munsell.

Stratigraphical structure of core 32A. X-ray radioscopy (negative) and tonalities according to Munsell Color Chart. 


\section{Concentración de metales en el sedimento}

La concentración media de $\mathrm{Cr}$ fue 26 ppm (rango de 11 a $49 \mathrm{ppm}$ ), la media de $\mathrm{V}$ fue $134 \mathrm{ppm}$ (rango de 120 a $155 \mathrm{ppm}$ ), la media de Ni fue $40 \mathrm{ppm}$ (rango de 19 a 73 ppm), la media de Co fue $7 \mathrm{ppm}$ (rango de 0 a 17), la media de Mn fue $46 \mathrm{ppm}$ (rango de 29 a $73 \mathrm{ppm}$ ), la media de Cd fue 44 ppm (rango de 32 a 54), y la media de Mo fue 51 ppm (rango 45 a 60 ppm). En la Fig. 4 se muestran los perfiles de estos metales a lo largo del testigo 32A. En general, estos perfiles mostraron un aumento de la concentración hacia el sedimento superficial, siendo el de $\mathrm{Mn}$ el que mostró el más fuerte aumento. No se identificó ninguna relación entre la estratigrafía del testigo y las variaciones de la concentración de los metales medidos.

\section{Caracterización de la materia orgánica}

La concentración de MOS en el testigo 32A fluctuó entre $19,9 \mathrm{mg} \mathrm{g}^{-1}$ y $39,9 \mathrm{mg} \mathrm{g}^{-1}$, con una media de 27,9 $\mathrm{mg} \mathrm{g}^{-1}$. Esto indica que el porcentaje medio de MOS contenido en el sedimento del testigo $32 \mathrm{~A}$ fue $2,7 \%$.

Los principales grupos funcionales detectados en los espectros de infrarrojo (IR) de la MOS y su fracción de hidrocarburos saturados a lo largo del testigo son entregados en las Tablas I y II. En general todos los espectros de MOS se caracterizaron por presentar los mismos grupos funcionales (Fig. 5), a excepción de los intervalos entre $0-4$ y $32-36 \mathrm{~cm}$, que no presentaron la banda atribuida a grupos $\mathrm{O}-\mathrm{H}$ (alcoholes o fenoles). Sin embargo, estas dos últimas muestras presentan bandas atribuibles a grupos funcionales del tipo $\mathrm{C}-\mathrm{O}$, las que en ausencia de grupos funcionales $\mathrm{O}-\mathrm{H}$, puede atribuirse a ésteres $(\mathrm{C}(=\mathrm{O})-\mathrm{O})$ o éteres $(\mathrm{C}-\mathrm{O}-\mathrm{C})$ (Tabla I). De igual manera, los espectros de IR obtenidos para la fracción de hidrocarburos saturados presentaron características muy similares a lo largo del testigo, respecto del tipo de grupos funcionales observados (Fig. 6).

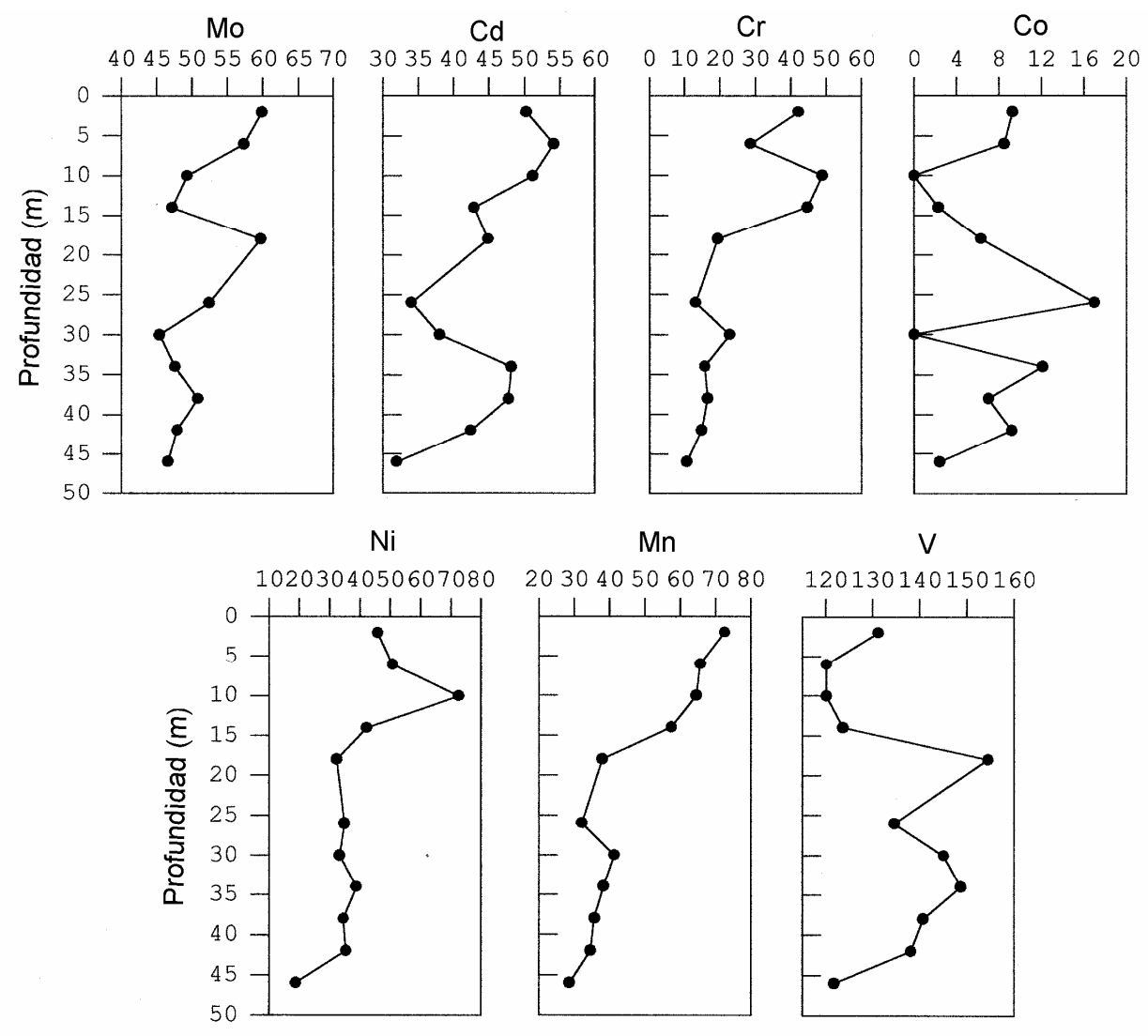

Figura 4

Perfiles de metales en muestras de $\mathbf{4} \mathrm{cm}$ correspondientes al testigo $32 \mathrm{~A}$.

Profiles of metals in 4-cm thick samples in core 32A. 
Tabla 1

Principales grupos funcionales detectados por espectroscopía infraroja con transformador de Fourier (IRTF) en la MOS del testigo 32A.

Main functional group detected by Fourier Transformed Infrared spectroscopy (FTIR) in the Soluble Organic Matter (SOM) of core 32A.

\begin{tabular}{|c|c|c|}
\hline $\mathrm{N}^{\circ}$ Banda & Longitud de Onda & Asignación \\
\hline 1 & $\approx 2960 \mathrm{~cm}^{-1}$ & Vibraciones de alargamiento de grupos metilos $\left(-\mathrm{CH}_{3}\right)$ de n-alcanos. \\
\hline 2 & $\approx 2850 \mathrm{~cm}^{-1}$ & Vibraciones de alargamiento de grupos metilenos $\left(-\mathrm{CH}_{2}-\right)$ de n-alcanos. \\
\hline 3 & $\approx 1460 \mathrm{~cm}^{-1}$ & Vibraciones de flexión de grupos metilos $\left(-\mathrm{CH}_{3}\right)$ de n-alcanos. \\
\hline 4 & $\approx 1375 \mathrm{~cm}^{-1}$ & Vibraciones de flexión de grupos metilenos $\left(-\mathrm{CH}_{2}\right)$ de n-alcanos. \\
\hline 5 & $\approx 720 \mathrm{~cm}^{-1}$ & Aromáticos monosustituidos o 1,2-disustituidos $\left(\approx 700\right.$ a $\left.750 \mathrm{~cm}^{-1}\right)$ \\
\hline & & $\begin{array}{l}\text { Vibraciones de oscilación de grupos metilenos }\left(\mathrm{\rho CH}_{2}\right) \text {. Esta banda es típica de n-alcanos } \\
\text { de mas de siete átomos de carbono, lo que indica la presencia de n-alcanos de cadena } \\
\text { recta. Esta banda puede estar solapada por la banda anterior correspondiente a } \\
\text { hidrocarburos aromáticos que es de mayor intensidad. }\end{array}$ \\
\hline 6 & $\approx 3100 \mathrm{~cm}^{-1}$ & $\begin{array}{l}\text { Vibraciones de alargamiento hidrógeno-carbono }(\mathrm{H}-\mathrm{C}) \text { de olefinas (alquenos). } \\
\mathrm{H}-\mathrm{C}=\mathrm{C} \text {. }\end{array}$ \\
\hline 7 & $\approx 1650 \mathrm{~cm}^{-1}$ & $\begin{array}{l}\text { Vibraciones de alargamiento carbono-carbono de olefinas (alquenos). } \\
\mathrm{C}=\mathrm{C} \text {. }\end{array}$ \\
\hline 8 & $\approx 650 \mathrm{~cm}^{-1}$ & $\begin{array}{l}\text { Vibraciones de flexión carbono-hidrógeno de olefinas (alquenos). } \\
\text { H-C }=\text { C. }\end{array}$ \\
\hline 9 & $\approx 900 \mathrm{~cm}^{-1}$ & Vibraciones hidrógeno-carbono en anillos aromáticos. \\
\hline 10 & $\begin{array}{l}\approx 1300 \mathrm{~cm}^{-1} \\
\approx 1410 \mathrm{~cm}^{-1}\end{array}$ & Vibraciones carbono-carbono (C-C) de anillos aromáticos. \\
\hline 11 & $\approx 3375 \mathrm{~cm}^{-1}$ & Vibraciones de alargamiento - $\mathrm{OH}$ de alcoholes y fenoles. \\
\hline 12 & $\approx 1260 \mathrm{~cm}^{-1}$ & $\begin{array}{l}\text { Esta banda puede ser asignada a los siguientes grupos funcionales: } \\
\text { 1.- Vibraciones C-O de alcoholes o fenoles. } \\
\text { 2.- Vibraciones de alargamiento C-O-C de éteres. } \\
\text { 3.- Vibraciones de alargamiento C-O de ésteres. }\end{array}$ \\
\hline 13 & $\approx 1738 \mathrm{~cm}^{-1}$ & $\begin{array}{l}\text { Vibraciones de alargamiento } \mathrm{C}=\mathrm{O} \text {. Esta banda puede ser asignada a los siguientes grupos } \\
\text { funcionales: ácidos carboxílicos, ésteres, aldehidos o cetonas. }\end{array}$ \\
\hline 14 & $\approx 1076 \mathrm{~cm}^{-1}$ & Vibraciones de alargamiento $\mathrm{C}-\mathrm{H}$ fuera del plano en hidrocarburos aromáticos. \\
\hline 15 & $\approx 1138 \mathrm{~cm}^{-1}$ & \\
\hline
\end{tabular}

Tabla 2

Principales grupos funcionales detectados por espectroscopía IRTF en la fracción de hidrocarburos saturados del testigo 32A.

Main functional group detected by FTIR spectroscopy in the saturated hydrocarbures fraction of core 32A.

\begin{tabular}{|c|c|c|}
\hline $\mathrm{N}^{\circ}$ Banda & Longitud de Onda & Asignación \\
\hline 1 & $\approx 3000 \mathrm{~cm}^{-1}$ & Vibraciones de alargamiento $\mathrm{C}-\mathrm{H}$ de cicloalcanos. \\
\hline 2 & $\approx 2960 \mathrm{~cm}^{-1}$ & Vibraciones de alargamiento de grupos metilos $\left(-\mathrm{CH}_{3}\right)$ de n-alcanos. \\
\hline 3 & $\approx 2850 \mathrm{~cm}^{-1}$ & Vibraciones de alargamiento de grupos metilenos $\left(-\mathrm{CH}_{2}-\right)$ de n-alcanos. \\
\hline 4 & $\approx 1461 \mathrm{~cm}^{-1}$ & Vibraciones de flexión de grupos metilos $\left(-\mathrm{CH}_{3}\right)$ de n-alcanos. \\
\hline 5 & $\approx 1384 \mathrm{~cm}^{-1}$ & Vibraciones de flexión de grupos metilenos $\left(-\mathrm{CH}_{2}\right)$ de n-alcanos. \\
\hline 6 & $\approx 723 \mathrm{~cm}^{-1}$ & $\begin{array}{l}\text { Aromáticos monosustituidos o 1,2-disustituidos }\left(\approx 700 \text { a } 750 \mathrm{~cm}^{-1}\right) \text {. } \\
\text { Vibraciones de oscilación de grupos metilenos }\left(\mathrm{CCH}_{2}\right) \text {. Esta banda es típica de n-alcanos } \\
\text { de mas de siete átomos de carbono, lo que indica la presencia de n-alcanos de cadena } \\
\text { recta. Esta banda puede estar solapada por la banda anterior correspondiente a } \\
\text { hidrocarburos aromáticos que es de mayor intensidad. }\end{array}$ \\
\hline 7 & $\approx 1246 \mathrm{~cm}^{-1}$ & Vibraciones de alargamiento carbono-carbono $(\mathrm{C}-\mathrm{C})$ de hidrocarburos aromáticos. \\
\hline 8 & $\approx 1292 \mathrm{~cm}^{-1}$ & \\
\hline 9 & $\approx 1338 \mathrm{~cm}^{-1}$ & \\
\hline 10 & $\approx 1061 \mathrm{~cm}^{-1}$ & Vibraciones de alargamiento carbono-hidrógeno de hidrocarburos aromáticos. \\
\hline 11 & $\approx 1138 \mathrm{~cm}^{-1}$ & \\
\hline 12 & $\approx 892 \mathrm{~cm}^{-1}$ & Vibraciones hidrógeno-carbono (H-C) en anillo aromáticos. \\
\hline
\end{tabular}




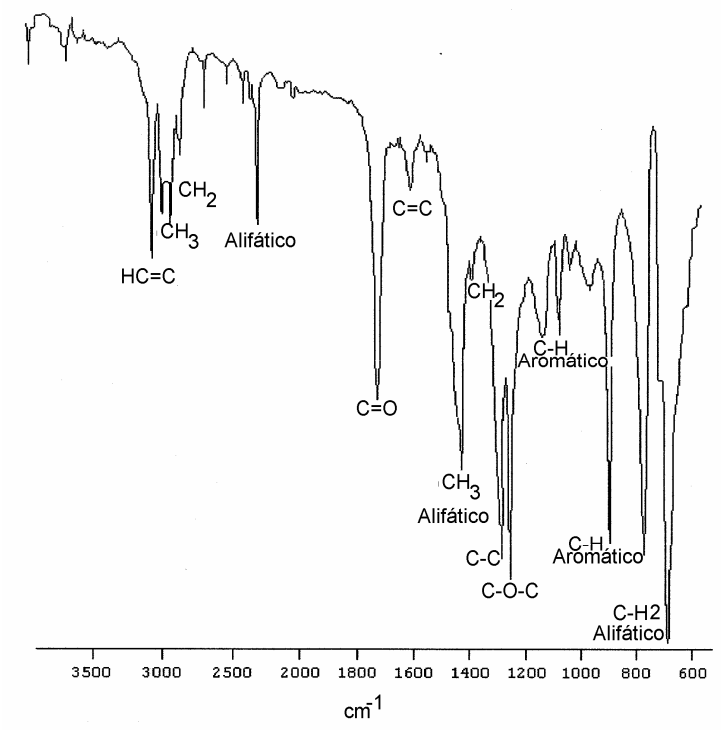

Figura 5

Espectro IR de MOS. Este espectro resume las características de los 5 análisis realizados en la MOS del testigo $32 \mathrm{~A}$.

Infrared spectra of Soluble Organic Matter (SOM). This spectra show the main characteristics of five analyses conducted on the SOM of core 32A.

La intensidad de las bandas atribuidas a grupos $\mathrm{CH}_{3}$ y $\mathrm{CH}_{2}$ (Figs. 5 y 6 ) sugiere la presencia de alcanos de cadena recta o ramificados. Esta observación junto con la presencia de la banda atribuida a $\rho \mathrm{CH}_{3}$ (a aproximadamente $720 \mathrm{~cm}^{-1}$ ), indicaría la existencia de alcanos de cadena recta (n-alcanos) de mas de 7 átomos de carbono. La presencia de n-alcanos de mas de 7 átomos de carbono es confirmada por la presencia de las bandas atribuidas a grupos $\mathrm{CH}_{3}, \mathrm{CH}_{2}$ y $\rho_{\mathrm{CH}}$ en los espectros IR de la fracción de hidrocarburos saturados. Por otra parte, se observa la presencia de olefinas en los espectros de IR de la MOS, lo que puede relacionarse a que estos alquenos se encuentran asociados a la fracción aromática o a la polar, probablemente formando parte de estas estructuras como cadenas insaturadas entre otros tipos de grupos funcionales como compuestos cíclicos insaturados.

Otra característica observada en la fracción de hidrocarburos saturados es la ausencia de bandas que se atribuyen a grupos carbonilos $(\mathrm{C}=\mathrm{O})$. Lo anterior indica que los grupos funcionales con oxígeno están asociados a las fracciones de mayor peso molecular de la MOS (hidrocarburos aromáticos o compuestos polares), e indirectamente descarta la presencia de cetonas o ésteres alifáticos. La ausencia de grupos funcionales con oxigeno en la fracción de hidrocarburos saturados, puede relacionarse a una reducción de estos grupos

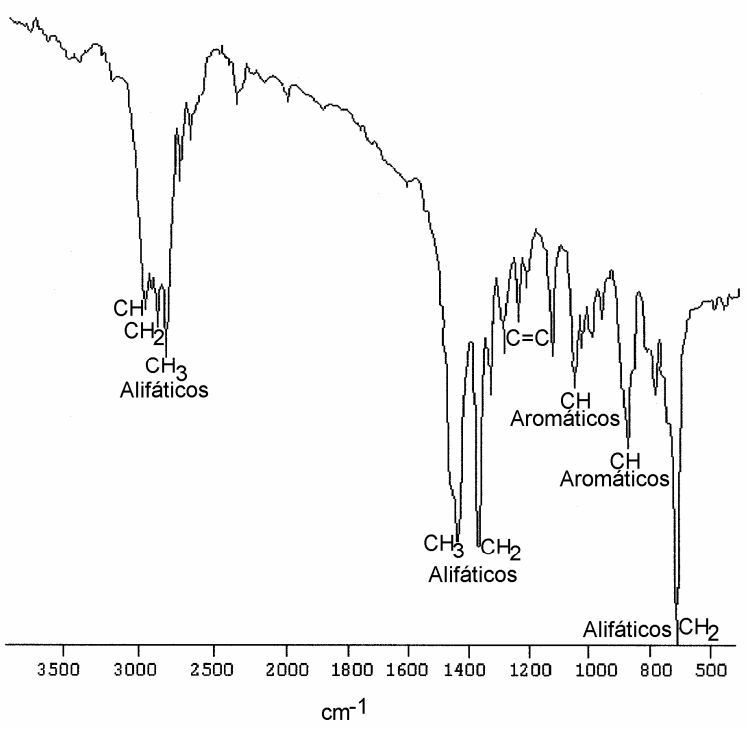

Figura 6

Espectros IR de hidrocarburos saturados. Este espectro resume las características de los 5 análisis realizados en la fracción de hidrocarburos saturados del testigo $32 \mathrm{~A}$.

Infrared spectra of saturated hydrocarbures. This spectra show that main characteristics of five analyses conducted on the saturated hydrocarbures fraction of core $32 \mathrm{~A}$.

funcionales, debido a las condiciones reductoras de este ambiente de sedimentación.

La presencia de bandas atribuidas a grupos funcionales aromáticos en la fracción de hidrocarburos saturados, puede ser consecuencia de contaminación con la fracción aromática de la MOS durante la separación por cromatografía de columna. Sin embargo, no se puede descartar la posibilidad de que existan anillos aromáticos simples enlazados a n-alcanos o cicloalcanos, lo cual puede asociarse a la presencia de bandas atribuidas a hidrocarburos aromáticos monosustituidos o 1,2-disustituidos, asociado a la banda intensa observada entre los 700 a $750 \mathrm{~cm}^{-1}$.

\section{Discusión}

\section{Condiciones de sedimentación durante el último milenio}

Los sedimentos de la bahía Mejillones presentan todas las características que han sido descritas como propias de ambientes de sedimentación pobres en oxígeno. Por ejemplo, Schrader (1992), Thunell et al. (1992), Lange \& Berger (1993) y Dean et al. (1994) indican que los ambientes reductores, pobres en oxígeno, limitan el desarrollo de la fauna bentónica $\mathrm{y}$, por lo tanto, favorecen la formación de sedimentos laminados. 
Ramorino \& Muñiz (1970) y Zúñiga et al. (1983) han indicado que en las zonas más profundas de la bahía Mejillones la macrofauna bentónica está ausente, situación que favorece una buena preservación de la estructura sedimentaria. De esta manera, la presencia de láminas, como una característica predominante del testigo 32A, es una clara evidencia de que durante el tiempo de sedimentación que éste abarca, el ambiente de fondo de la bahía era pobre en oxígeno. Otros testigos que abarcaron los últimos 3000 años también mostraron una estructura laminar (Ortlieb et al. 2000; Valdés 1998), lo que indicaría que esta característica se ha mantenido durante varios milenios.

Además de las características estratigráficas la condición redox también puede ser inferida a partir del registro de indicadores geoquímicos. Por ejemplo, una serie de metales como $\mathrm{Cr}, \mathrm{Cu}, \mathrm{Ni}, \mathrm{Mo}, \mathrm{Cd}$ y V se encuentran notablemente concentrados en sedimentos en donde se generan condiciones sulfato-reductoras próximas al fondo marino (Didyk et al. 1978; Malcolm 1985; van Geen et al. 1995; Calvert et al. 1996; Dean et al. 1997). En el caso de Mejillones, los valores de Cd y Mo por ejemplo, encontrados a lo largo del testigo, pueden ser considerados como elevados (Valdés 1998), y por lo tanto, reflejarían también, el predominio de condiciones subóxicas/anóxicas durante el pasado.

Por otra parte, Jones \& Manning (1994) evaluaron una serie de índices de metales traza medidos en formaciones de rocas sedimentarias Jurásicas del Mar del Norte, Noruega y la costa de Inglaterra, que pueden ser utilizados para estudiar la oxigenación de ambientes marinos en el pasado. Uno de los índices evaluados fue $\mathrm{V} / \mathrm{Cr}$. El $\mathrm{Cr}$ se asocia al detritus clástico del sedimento, en donde puede sustituir al $\mathrm{Al}$ en las arcillas, puede ser readsorbido, o presentarse como Cromita (Jones \& Manning 1994). Por su parte, el V puede estar asociado a la materia orgánica por la incorporación de $\mathrm{V}^{+4}$ en las porfirinas, y así ser concentra-do en sedimentos depositados bajo condiciones reductoras (Jones \& Manning 1994). Calvert et al. (1996) indican que estos metales son transformados a su valencia más baja en condiciones reduc-toras, y son removidos hacia los sedimentos como óxidos o hidróxidos insolubles. Estudios anteriores (Ernst 1970; Krecji-Graf 1974) indicaban que valores de la relación $\mathrm{V} / \mathrm{Cr}$ superiores a 2 representaban condiciones de depositación anóxicas, con $\mathrm{H}_{2} \mathrm{~S}$ presente en el agua adyacente al fondo marino; valores menores a 2 indicaban aguas un poco más oxigenadas; y valores cercanos a 1 sugerían que la interface óxica/anóxica se encontraba ubicada dentro de la columna de sedimento. Sin embargo, basado en sus observaciones Jones \& Manning (1994) plantearon que valores superiores a 2 representan condiciones de depositación disóxicas, y que valores superiores a 4,25 reflejan condiciones subóxicas/anóxicas.

Otro índice evaluado por Jones \& Manning (1994) fue $\mathrm{Ni} / \mathrm{Co}$. Este índice puede ser usado como un indicador redox ya que en los sedimentos marinos ambos metales se encuentran en la pirita, mineral abundante en ambientes sulfato reductores. Se había reportado que valores altos $(>1)$ evidenciaban condiciones reductoras en el ambiente de sedimentación. Sin embargo, los autores propusieron una separación más precisa. Así, valores mayores a 5 reflejarían condiciones de sedimentación disóxicas y valores mayores a 7 reflejarían condiciones subóxicas/anóxicas.

La Fig. 7 presenta los perfiles obtenidos del cálculo

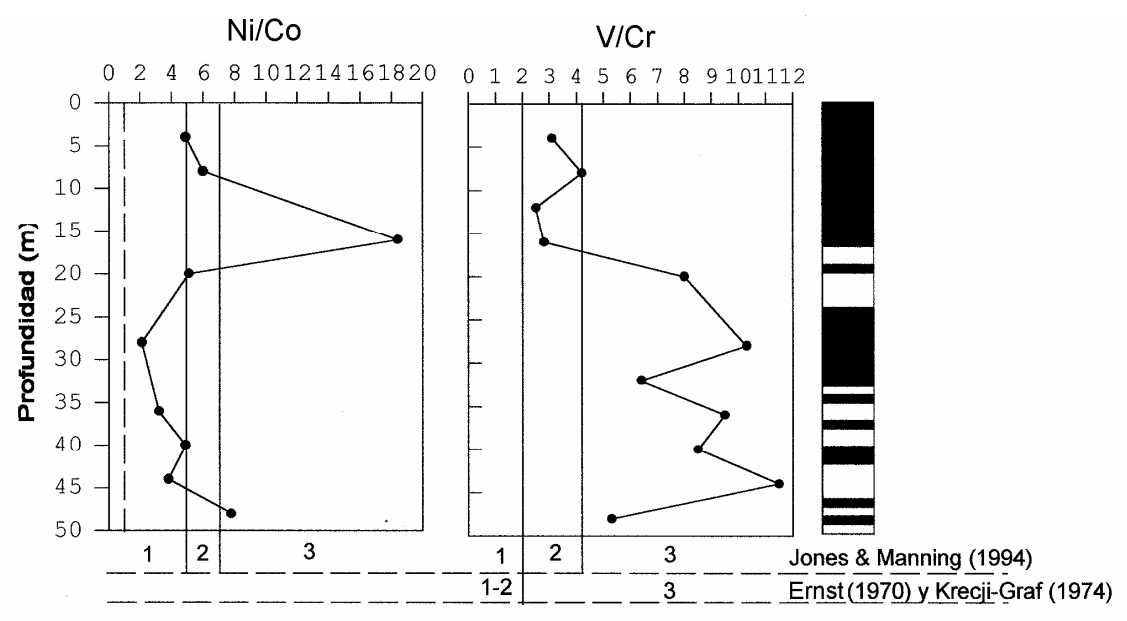

Figura 7

Indices $\mathrm{Ni} / \mathrm{Co}$ y $\mathrm{V} / \mathrm{Cr}$ en el testigo 32A. Se indica la clasificación de diferentes autores para cada índice: 1 es óxico, 2 es disóxico, y 3 es subóxico/anóxico. La línea discontinua en $\mathrm{Ni} / \mathrm{Co}$ corresponde a la relación que tiene este índice en la pirita, típica de ambientes reductores (Jones \& Manning, 1994). La terminología empleada referente a la oxigenación corresponde a Tyson \& Pearson (1991).

$\mathrm{Ni} / \mathrm{Co}$ and $\mathrm{V} / \mathrm{Cr}$ ratios in core $32 \mathrm{~A}$. Every ratio is indicated according to several authors: 1 is oxic, 2 is dysoxic, 3 is suboxic/anoxic. The discontinuous line $(\mathrm{Ni} / \mathrm{Co})$ corresponds to the relationship between this ratio and pyrite, that is typical of reducing environment (Jones \& Manning 1994). Terminology used for the degree of oxygenation is taken from Tyson \& Pearson (1991). 
de ambos índices en el testigo 32A. Si se analiza la evolución del ambiente de fondo de la bahía sobre la base de estos resultados, pareciera apreciarse algunas contradicciones. Por una parte, el índice $\mathrm{V} / \mathrm{Cr}$ sugiere que el fondo de la bahía ha transitado hacia el presente, desde una condición subóxica/anóxica a una disóxica. Mientras que el índice Ni/Co muestra una transición desde un ambiente óxico hacia uno subóxico/anóxico. Jones Manning (1994) también encontraron discrepancias entre los resultados de los diferentes índices utilizados por ellos con los reportados por otros autores, concluyendo que se necesita una combinación de antecedentes tanto geoquímicos como paleoecológicos para identificar los mejores índices geoquímicos que caracterizan a un ambiente de depositación. Por otra parte, Ernst (1970) indica que factores como el tamaño de grano y el contenido de carbonatos de los sedimentos pueden alterar los valores de los índices de paleoxigenación. Es posible, además, que los resultados obtenidos en el caso de la bahía Mejillones se deban a que se trata de sedimentos no consolidados y de edad notoriamente más reciente a aquellos utilizados por Jones \& Manning (1994).

Sin duda que la estructura laminar de los sedimentos de la bahía Mejillones es la evidencia más fuerte que respalda la idea de que el oxígeno disuelto de las aguas subsuperficiales nunca ha alcanzado niveles suficientemente altos como para permitir el desarrollo de macrofauna bentónica desde que se depositaron estos sedimentos. Esta situación tiene una buena correspondencia con la clasificación de Ernst (1970) y Krecji-Graf (1974), según la cual el fondo de la bahía se ha mantenido durante todo el último milenio bajo una condición de anoxia.

En Mejillones, otros estudios (Ortlieb et al. 1994; 2000) han utilizado los bioindicadores presentes en testigos de hasta un metro de longitud y recuperados en diferentes partes de la bahía, para inferir condiciones de oxigenación subsuperficiales durante los últimos milenios. Estos estudios mostraron la presencia de comunidades de foraminíferos bentónicos, de entre los cuales Bolivina seminuda, que es capaz de vivir en ambientes con muy escaso oxígeno (Kahio 1994), fue encontrado a lo largo de toda la columna de sedimento. Sin embargo, en algunos estratos, con una marcada disminución del número de ejemplares de $B$. seminuda, se observó un aumento de otras especies de foraminíferos bentónicos como Buliminella elegantissima, Nonionella auris y Nonionella pulchella. Los autores plantearon que durante estos períodos se produjo un aumento del oxígeno de fondo en la bahía, con un predominio de condiciones disóxicas. Sin embargo, la ausencia de estructuras de bioturbación en el testigo 32A es una evidencia de que este aumento de la disponibilidad de oxígeno no fue suficiente como para permitir el desarrollo de macrofauna bentónica, principal responsable de la pérdida de la estructura primaria de los sedimentos marinos (Reineck \& Singh, 1980). Así, las posibles variaciones de la oxigenación durante el pasado reciente no parecen haber cambiado fuertemente la condición de pobreza de oxígeno predominantes en el ambiente de fondo de la bahía.

\section{Preservación de materia orgánica}

La concentración de MOS registrada en el testigo 32A (27,9 $\mathrm{mg} \mathrm{g}^{-1}$ en promedio) corresponde sólo a la fracción más pequeña del contenido total de materia orgánica de los sedimentos. La otra fracción, mayoritaria, corresponde al kerógeno o materia orgánica insoluble, que junto con la fracción anterior constituyen el total de materia orgánica de los sedimentos recientes (Duran 1973). Sin embargo, la concentración de MOS del testigo estudiado es un buen indicador de la alta productividad biológica del sector, ya que es notoriamente más alta que la registrada en otros sistemas, como por ejemplo, en los sedimentos anóxicos de la Cuenca de Cariaco (Venezuela) en donde se han medido valores promedio de 750 ppm (López \& Lo Mónaco, resultados no publicados).

La ausencia de grupos carbonilos en la fracción de hidrocarburos saturados de la MOS, se relaciona a ambientes carbonáticos muy reductores, como consecuencia de la reducción de n-ácidos grasos (Tissot \& Welte 1978). Esta ausencia de bandas atribuidas a grupos carbonilos en la fracción saturada del testigo $32 \mathrm{~A}$ es consistente con la reducción de los n-ácidos grasos, lo cual es una reacción común durante la diagénesis de la materia orgánica en ambientes sedimentarios reductores (Tissot \& Welte, 1984).

La presencia de hidrocarburos insaturados (alquenos), los cuales están presentes en los organismos vivos (Tissot \& Welte 1984), junto con la presencia de la banda atribuida al grupo carbonilo $(\mathrm{C}=\mathrm{O})$ (aproximadamente a $1700 \mathrm{~cm}^{-1}$ ) en la MOS (Fig. 5, Tabla I) del testigo 32A, son evidencias de los pocos cambios postsedimentarios de la materia orgánica. Sin embargo, para la fracción de hidrocarburos saturados de la MOS estas dos bandas están ausentes (Fig. 5, Tabla II). En este sentido Tissot \& Welte (1984) plantean que una disminución de la banda del grupo carbonilo, en muestras de materia orgánica, se debe a la eliminación progresiva de este grupo funcional. De igual manera, durante la diagénesis de la materia orgánica ocurre una disminución de los grupos funcionales de alquenos $(\mathrm{C}=\mathrm{C})$, como consecuencia de hidrogenación que lleva a la formación de compuestos saturados. Así, la presencia de estos grupos funcionales en la MOS de las muestras de sedimento a lo largo del testigo 32A, que representa 1000 años de sedimentación, permiten 
concluir que los compuestos orgánicos han sufrido una mínima degradación en el sedimento.

La ausencia de enlaces del tipo alqueno o carboxílico en la fracción saturada puede estar indicando que esta fracción, más susceptible a modificaciones durante la diagénesis de la materia orgánica depositada en sedimentos marinos, sí ha sufrido cambios, los que corresponderían a hidrogenación y reducción de los ácidos grasos. Estas características son evidencias de procesos diagenéticos (Tyssot \& Welte, 1978).

Otro ejemplo de cambios diagenéticos de la MOS es la presencia de bandas atribuidas a grupos funcionales de hidrocarburos aromáticos monosustituidos o 1,2disustituidos, la que podría estar relacionada a procesos de aromatización de hidrocarburos saturados durante la diagénesis de la materia orgánica.

Basado en las características de los espectros de IR a lo largo del testigo 32A, donde se observa una abundancia relativa de grupos funcionales del tipo $\mathrm{C}=\mathrm{O}$ (carbonilo) y $\mathrm{C}=\mathrm{C}$ (alquenos), se sugieren pocos cambios postsedimentarios en la materia orgánica ya que estas conservan algunas características de sus precursores biológicos. Sin embargo, la ausencia de grupos alquenos y carbonilo en la fracción saturada de la MOS, indica que esta fracción la cual es más susceptible a cambio diagenéticos, sí ha sido alterada en los sedimentos.

En general, y de acuerdo a los resultados de estos análisis es posible indicar que la materia orgánica evidencia un bajo grado de madurez, lo cual refuerza la hipótesis de que la preservación de los compuestos orgánicos en los sedimentos de la bahía Mejillones, es altamente eficiente desde el momento mismo en que éstos alcanzan el fondo marino.

\section{Conclusiones}

El estudio combinado de metales y de materia orgánica soluble contenidos en un testigo de sedimentos de la bahía Mejillones del Sur ha permitido identificar las condiciones de oxigenación y preservación de materia orgánica durante el último milenio.

Los sedimentos acumulados en la bahía Mejillones del Sur muestran claras evidencias del predominio de condiciones subóxicas/anóxicas en el ambiente de depositación, al menos, durante el último milenio. Tanto las características sedimentológicas como geoquímicas del testigo 32A permiten postular que el ambiente de fondo de la bahía se ha caracterizado por la presencia de una capa anóxica que comienza varios metros sobre el piso marino.
Los estudios realizados con bioindicadores sobre sedimentos coetaneos del testigo 32A, mostraron un aumento de la abundancia de foraminíferos bentónicos que fue interpretado como el desarrollo de aguas de fondo un poco más oxigenadas (probablemente $>0,3$ $\mathrm{ml} \cdot \mathrm{l}^{-1}$ ). Sin embargo se debe considerar que estos foraminíferos constituyen una microfauna que vive en la parte superficial de la columna de sedimento (interfase), de tal manera que un aumento de la disponibilidad de oxígeno en esta zona y que propicie el desarrollo de estos organismos, no debiera comprometer la preservación de la estructura sedimentaria vía mecanismo de bioturbación. Por tal motivo, parece más conveniente indicar que la presencia de láminas como característica común en los sedimentos de Mejillones sea interpretada como un reflejo de condiciones pobres de oxígeno en el ambiente subsuperficial, más que como una evidencia de anoxia estricta.

De esta forma, tanto la evidencia geoquímica (metales e índice de metales), como la biológica (foraminíferos bentónicos) y estratigráficas (sedimentos laminados), representan condiciones de depositación pobres en oxígeno (probablemente fluctuando entre anóxica y disóxica), durante los últimos milenios en la bahía Mejillones.

El análisis IRTF realizado a distintos niveles de profundidad en el testigo $32 \mathrm{~A}$, puso en evidencia que se han registrado pocos cambios postdepositacionales de la materia orgánica soluble. La presencia de los mismos grupos funcionales presentes en los espectros obtenidos de las muestras de este testigo es interpretada como una evidencia de que en el transcurso de los pasados mil años la diagénesis temprana ha afectado en grado mínimo a los constituyentes orgánicos del sedimento. Esta conclusión también es apoyada por el hecho de que los grupos funcionales normalmente presentes en los precursores biológicos (organismos vivos) son los mimos que se encontraron en la materia orgánica soluble del testigo $32 \mathrm{~A}$.

Por su parte, las evidencias de cambios diagenéticos detectados en la fracción de hidrocarburos saturados (más susceptible a alteraciones), son mínimas y propias de ambientes reductores.

El alto contenido de materia orgánica y su buen estado de preservación tienen relación directa con la pobreza de oxígeno que han presentado las aguas subsuperficiales de la bahía, durante el último milenio.

\section{Agradecimientos}

Los autores desean agradecer a Roxana Infante y Eduardo Labastidas por su colaboración en el tratamiento de las muestras, y al Dr. Alexis Lira por su apoyo en el análisis de los metales. 
El autor principal fue beneficiado por una beca del IRD para realizar una estadía de investigación en el Instituto de Ciencias de la Tierra de la Universidad Central de Venezuela, lugar en donde se realizaron los análisis.

Finalmente se desea agradecer los comentarios y correcciones de dos revisores anónimos que ayudaron a mejorar este manuscrito.

\section{Literatura Citada}

Alongi D, S Boyle, F Tirende \& C Payn. 1996. Composition and behavior of trace metals in post-oxic sediments of the gulf of Papua, Papua New Guinea. Estuarine Coastal Shelf Science 42(2): 197-212.

Brown J, A Colling, D Park, J Phillips, D Rothery \& J Wright. 1994. Ocean Chemistry and Deep-Sea sediments, 133 p. The Open University/Pergamon, London.

Calvert S, R Bustin \& E Ingall. 1996. Influence of water column anoxia and sediment supply on the burial and preservation of organic carbon in marine shales. Geochimica et Cosmochimica Acta 60(9): 1577-1593.

Dean W, J Gardner \& R Anderson. 1994. Geochemical evidence for enhanced preservation of organic matter in the oxygen minimum zone of the continental margin of northern California during the late Pleistocene. Paleoceanography 9(1): 47-61.

Dean W, J Gardner \& D Piper. 1997. Inorganic geochemical indicators of glacial-interglacial changes in productivity and anoxia on the California continental margin. Geochimica et Cosmochimica Acta 61(21): 45074518.

Didyk B, B Simoneit, S Brassell \& G Eglinton. 1978. Organic geochemical indicators of paleoenvironmental conditions of sedimentation. Nature 272 (5660): 216-222.

Durand B. 1973. Sedimentary organic matter and kerogen. Definition and quantitative importance of kerogen, 13-33. En: KEROGEN, Insoluble organic matter from sedimentary rocks. Technip, Paris.

Ernst T. 1970. Geochemical facies analysis. 152 p. Elsevier Press, Amsterdam.

Gallardo V. 1992. Presencia de material orgánico teñido por metales en los fondos de Thioploca de la plataforma continental frente a Bahía de Concepción, Chile. Gayana Oceanológica 1: 27-33.

Hulthe G, S Hulth \& P Hall. 1998. Effect of oxygen on degradation rate of refractory and labile organic matter in continental margin sediments. Geochimica et Cocmochimica Acta 62 (8): 1319-1328.

Jones B, \& D Manning. 1994. Comparison of geochemical indices used for the interpretation of paleoredox conditions in ancient mudstones. Chemical Geology 111: 111-129.

Kaiho K. 1994. Benthic foraminiferal dissolve-oxygen index and dissolved-oxygen levels in the modern ocean. Geology 22: 719-722.

Kamykowski D \& S Zentara. 1990. Hypoxia in the world as recorded in the historical data set. Deep-Sea Research 37(12): 1861-1874.

Kemp A, R Pearce \& J Baldauf. 1993. Neogene El Niño/Southern Oscillation records in laminated diatom ooze from the Eastern Equatorial Pacific. EOS 74(43): 372.

Krecji-Graf K. 1975. Geochemical facies os sediments. Soil Science 119: 20-23.

Lallier-Verges E, P Bertrand, A Huc, D Buckel \& P Tremblay. 1993. Control of the preservation of organic matter by productivity and sulphate reduction in Kimmeridgian shales from Dorset (UK). Marine and Petroleum Geology 10: 600-605.

Lange C. \& W Berger. 1993. Paleoclimatic significance of Santa Barbara laminated sediments: A history of upwelling and El Niño Events. EOS 74(3): 372.

Libes S. 1992. An introduction to marine biogeochemistry, 289 p. John Wiley \& Sons, Inc.

Malcolm S. 1985. Early diagenesis of molybdenum in estuarine sediments. Marine Chemistry 16: 213-225.

Murray J \& K Kuivila. 1990. Organic matter diagenesis in the northeast Pacific: transition from aerobic red clay to suboxic hemipelagic sediments. Deep-Sea Research 37(1): 59-80.

Ortlieb L, O Zuñiga, R Follegati, R Escribano, I Kong, L Rodriguez, Ph Mourguiart, J Valdes \& P Iratchet. 1994. Paleoceanografía de la Bahía de Mejillones del Sur (Antofagasta, Chile): Resultados preliminares para el último milenio. Estudios Oceanológicos 13: 45-55.

Ortlieb L, R Escribano, R Follegati, O Zuñiga, I Kong, L Rodriguez, J Valdes, P Iratchet \& N Guzmán. 2000. Ocean-climatic changes during the last 2,000 years in a hypoxic marine environment of Northern Chile $\left(23^{\circ} \mathrm{S}\right)$. Revista Chilena de Historia Natural 73: 221-242.

Pretsch E, T Clerc, J Seibl \& W Simon. 1989. Tablas para la elucidación estructural de compuestos orgánicos por métodos espectroscópicos, 307 p.. Editorial Alambra, Caracas.

Ramorino L \& L Muñiz. 1970. Estudio cuantitativo general sobre la fauna de fondo de la bahía de Mejillones. Revista de Biología Marina 14(2): 79-93.

Ransom B, D Kim, M Kastner \& S Wainwright. 1998. Organic matter preservation on continental slopes: Importance of mineralogy and surface area. Geochimica et Cocmochimica Acta 62(8): 1329-1345.

Reineck H \& I Singh. 1980. Depositional sedimentary environments. With reference to terrigenous clastics, 570 p. Springer-Verlag Press, Second Edition, New York.

Ridway Y \& N Price. 1987. Geochemical associations and post-depositional mobility of heavy metals in coastal sediments: Loch Etive, Scotland. Marine Chemistry 21: 
$229-248$.

Riley J \& R Chester. 1973. Introduction to marine chemistry, 397 p. Academic Press. London.

Schrader H. 1992. Comparison of Quaternary coastal upwelling proxies off central Peru. Marine Micropaleontology 19: 29-47.

Silverstein R, G Bassler \& T Morrill. 1980. Identificación espectrométrica de compuestos orgánicos, $353 \mathrm{p}$. Editorial Diana, Caracas.

Szefer P \& B Skwarzec. 1988. Distribution and possible source of some elements in the sediment cores of the southern Baltic. Marine Chemistry 23: 109-129.

Thunell R, M Oingmin, S Calvert \& F Pedersen. 1992. Glacial-Holocene biogenic sedimentation patterns in the south China Sea: Productivity variations and surface water $\mathrm{pCO}_{2}$. Paleoceanography 7(2): 143-162.
Tissot W \& D Welte. 1984. Petroleum Formation and Occurrence, 699 p. Springer-Verlag Press, Berlin.

Tyson R \& T Pearson. 1991. Modern and ancient continental shelf anoxia: an overwie, 58: 1-24. En: R. Tyson and T. Pearson (eds), Modern and ancient continental shelf anoxia. Geological Society, London.

VAN GEEN A, D Mccorkle \& G KLINKHAMMER. 1995. Sensitivity of the phosphate-cadmium-carbon isotope relation in the ocean to cadmium removal by suboxic sediments. Paleoceanography 10 (2): 159-169.

Valdés J. 1998. Evolución oceanográfica reciente de la Bahía Mejillones del Sur $\left(23^{\circ} \mathrm{S}\right)$. Evidencia geoquímica en sedimentos marinos, 114 p. Tesis Doctoral, Centro EULAChile Universidad de Concepción.

Zúñiga O, H Baeza \& $\mathbf{R}$ Castro. 1983. Análisis de la macrofauna bentónica del sublitoral de la bahía de Mejillones del Sur. Estudios Oceanológicos 3: 41-62. 